Chirurg 2011 $\cdot 82: 197-198$

DOI 10.1007/s00104-010-2009-0

Online publiziert: 23. Februar 2011

(c) Springer-Verlag 2011

\author{
C.M. Seiler · M.W. Büchler \\ Klinik für Allgemein-, Viszeral- und Transplantationschirurgie, \\ Universitätsklinikum Heidelberg
}

\title{
Chirurgisch bedingte Infektionen
}

lich eingesetzt werden, steigt. Längst sind nicht nur die Orthopäden, Unfallchirurgen oder Gefäßchirurgen Meister im Einbau von Materialien, die den Körper unterstützen sollen, aber eben auch das Risiko der Infektion beinhalten, auch die Allgemein- und Viszeralchirurgie hat erkannt, dass oft nur Fremdmaterialien auf Dauer eine Heilung ermöglichen. Bestes Beispiel auf diesem Gebiet ist die Hernienchirurgie: Wie viele Netze und Befestigungsmaterialien wurden hier in den letzten 20 Jahren entwickelt und leider oft ohne eine gute klinische Studie auf den Markt gebracht? Wer hat die mit diesen Fremdmaterialien assoziierten Infektionsraten bis hin zu Darmfisteln valide klinisch untersucht? Das Problem der Netzinfektion ist allgegenwärtig und wird deshalb auch in einem eigenen Beitrag von Dietz et al. kritisch beleuchtet.

An erster Stelle steht dabei das Grundverständnis der pathophysiologischen und molekularbiologischen Zusammenhänge; sie werden in einem hervorragenden Beitrag von Frau B. Vollmar dargestellt. Zentrum ist das Immunsystem des Menschen, das durch seine Komplexität fasziniert und heute besser denn je verstanden wird. Nicht das Hemmen von Mediatoren steht im Vordergrund, sondern die Unterstützung des Menschen in seiner Reaktion auf die Infektion. Medikamentösen Verfahren kommt deshalb auch in der Zukunft eine zentrale Bedeutung zu, denn stets reagiert bei der Infektion der ganze Mensch mit allen seinen Möglichkeiten.

Welche Rolle spielt die chirurgische Infektion? Der Anteil an Implantaten und sonstigen Fremdkörpern, die tagtäg-

\section{(- Das Problem der Netzinfektion ist allgegenwärtig}

Orthopädie und Unfallchirurgie stehen bei der periprothetischen Infektion vor großen Herausforderungen. Weder die zeitgerechte Diagnostik noch die adäquate Therapie können als geklärt angesehen werden. Der aktuelle Stand wird von Per$\mathrm{ka}$ et al. dargestellt und fordert geradezu nach objektiver klinischer Forschung. Bei Heilungsraten von $80 \%$ gibt es noch Raum für Verbesserungen, die kaum alleine, sondern nur gemeinsam erreicht werden können.

Positiv sind die evidenzbasierten Entwicklungen im Bereich der Antibiotikaprophylaxe. Durch konsequente For- schung in Form multizentrischer randomisierter Studien konnte hier eine Reduktion postoperativer Infektionen erreicht werden [2]. Jede Praxis und Klinik sollte basierend auf den verfügbaren Empfehlungen, die von Knebel et al. vorgestellt werden, ihre verbindlichen Prophylaxeaber auch Therapieschemata aktualisieren, um Infektionen gar nicht erst entstehen zu lassen. Diese Aufgabe ist sinnvoll nur interdisziplinär zu lösen und sollte die bei der Behandlung des Patienten involvierten Fachgebiete berücksichtigen.

Die Entwicklung von Strategien zur Vermeidung von Wundinfektionen ist eine gemeinsame Herausforderung für alle chirurgischen Fachgebiete. Wir haben gelernt Wundinfektionen zu definieren und damit eine Vergleichbarkeit von Infektionsraten erreicht [1]. Neben den pharmakologischen Interventionen sind auch die chirurgischen Aspekte nicht zu vernachlässigen. Wie und mit was wird das Operationsfeld gereinigt? Welche Rolle spielen beschichtete Implantate und Nahtmaterialien [3]? Nicht nur die Vermeidung, sondern auch die Therapie der Wundinfektion ist ein Thema, wie Maier et al. in ihrem Beitrag darstellen. Sind das chirurgische Débridement und die Sekundärnaht noch ein Thema in Zeiten der Vakuumverbände? Kostspielige und langwierige nicht operative Therapieverfahren müssen in randomisierten Studien ihre Nutzen- und Kosteneffektivität beweisen, bevor wir sie flächendeckend zum Einsatz empfehlen können.

Die Prinzipien bei der Behandlung der Peritonitis haben sich seit über 100 Jahren kaum verändert. An primärer Stelle steht 
die Herdsanierung, gefolgt von der Reinigung und der anschließenden supportiven Therapie [4]. Trotz aller Verbesserungen in Diagnostik und Therapie bleibt laut Strobel et al. die Letalität von bis zu 20\% hoch und aufgrund der demographischen Entwicklung mit älteren und multimorbiden Patienten werden die Anforderungen an uns wachsen. Das vom Bundesministerium für Bildung und Forschung geförderte Kompetenznetz Sepsis hat sich diesem Thema gestellt und führt bereits große multizentrische randomisierte Studien zur pharmakologischen Therapie durch [5].

Es ist an der Zeit, dass auch die ungeklärten Fragen der operativen Behandlung durch diesen Studientyp angegangen werden. Im Vordergrund unserer Bemühungen sollte die Prophylaxe stehen, denn jede Reduktion der jetzigen Infektionsraten wird von uns Chirurgen und den Kostenträgern begrüßt, der Patient wird sie aber ganz entschieden erwarten.
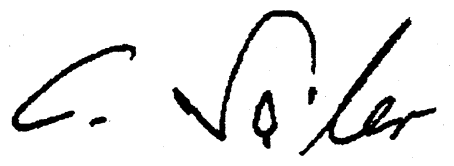

C.M. Seiler

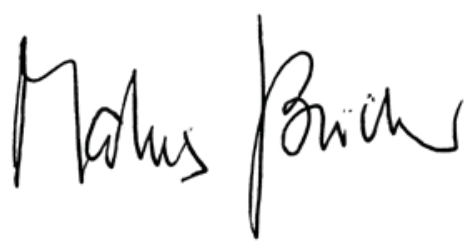

M.W. Büchler

\section{Korrespondenzadresse}

\section{Prof. Dr. M.W. Büchler}

Klinik für Allgemein-, Viszeral- und

Transplantationschirurgie, Universitätsklinikum Heidelberg, Im Neuenheimer Feld 110, 69120 Heidelberg

Markus.buechler@med.uni-heidelberg.de

\section{Literatur}

1. Gastmeier P, Brandt C, Sohr D et al (2004) [Surgical site infections in hospitals and outpatient settings. Results of the German nosocomial infection surveillance system (KISS)]. Bundesgesundheitsbl Gesundheitsforsch Gesundheitssch 47(4):339-344

2. Wacha H HU, Isenmann R, Kujath P et al (2010) Perioperative Antibiotikaprophylaxe. Chemother J 19:70-84
3. Tautenhahn J, Meyer F, Buerger T et al (2010) Interactions of neutrophils with silver-coated vascular polyester grafts. Langenbecks Arch Surg 395(2):143-149

4. De Waele JJ (2010) Early source control in sepsis. Langenbecks Arch Surg 395(5):489-494

5. Brunkhorst FM, Engel C, Bloos F et al (2008) German Competence Network Sepsis (SepNet). Intensive insulin therapy and pentastarch resuscitation in severe sepsis. N Engl J Med 358(2):125-139

\section{www.SpringerMedizin.de/chirurgie}

\section{Alles rund um Ihr Fachgebiet}

Wählen Sie ab sofort den direkten Weg zu News

und Fachbeiträgen aus Ihrem Fachgebiet:

Auf www.SpringerMedizin.de/chirurgie

halten wir Sie immer auf dem Laufenden.

Hier erwartet Sie ein umfassendes Informationsangebot:

- News aus Berufs- und Gesundheitspolitik

- Ausgewählte Meldungen und Beiträge aus Ihrem Fachgebiet

- Die wichtigsten Studien und Publikationen - für Sie zusammengefasst und kommentiert

- Kompetente Unterstützung bei schwierigen Fragestellungen durch unsere Expertenräte

- Medizinische Fortbildung in Form von Bilderstrecken, Podcasts bis hin zu Reviews und CME-Beiträgen aus den Fachzeitschriften von Springer Medizin

So einfach geht's: Nach einmaliger Registrierung steht Ihnen ein umfangreiches kostenloses Angebot zur Verfügung.

SpringerMedizin.de - Das Internet der Ärzte 